\title{
EOP's:
}

\section{Asma y reflujo gastroesofágico}

\begin{abstract}
Escenario clínico:
En un pase de pacientes ambulatorios un Residente de primer año comenta que atiende a un paciente de 25 años asmático desde la infancia. El paciente presentaba asma leve intermitente, pero este año se tornó moderada y con mala respuesta a la medicación. El Jefe de Residentes sugiere interrogar al paciente acerca de posibles desencadenantes ambientales, verificar la técnica de empleo de los aerosoles y si esto no explica el cuadro, descartar reflujo gastroesofágico ya que puede ser la causa de los síntomas. Un Residente de tercer año comenta que existe evidencia suficiente de la asociación entre reflujo gastroesofágico (RGE) y asma bronquial. Sin embargo no sabe si el tratamiento del reflujo mejora el asma.
\end{abstract}

\section{Preguntas que generó el caso:}

En los pacientes asmáticos con RGE (población), el tratamiento del RGE (intervención) mejora la función pulmonar o los síntomas del asma (resultados clínicos de interés)?

\section{Asma y reflujo gastroesofágico:}

El RGE es frecuente en adultos y niños con asma bronquial y se lo ha vinculado como factor de mal control del broncoespasmo. Los mecanismos mediante los cuales se propone que ocurre esto incluyen: aspiración de ácido o estimulación vagal que aumenta la hiperreactividad bronquial al estímulo exógeno de diferentes alergenos.

Pocos tópicos en la medicina son tan controvertidos como la relación entre el asma y el RGE. A pesar de la incertidumbre alrededor de esta relación, existe un fuerte vínculo entre ambas condiciones. La prevalencia de RGE en asmáticos es variable dependiendo de las definiciones que se empleen para ambas enfermedades y la población estudiada. Algunos estudios han mostrado que casi la mitad de los asmáticos presentan RGE comparado con el $10 \%$ de los no asmáticos.

En general, se recomienda buscar RGE en asmáticos (especialmente si el control es difícil) y de estar presente, indicar tratamiento para mejorar el control del asma. El tratamiento del RGE puede ser conservador con diversos fármacos o incluso llegar a ser quirúrgico.

Debemos señalar que muchos asmáticos presentan RGE muy sintomático y que requieren tratamiento independientemente del efecto sobre la función pulmonar. La duda"es si en pacientes asmáticos con RGE que no merece tratamiento per se, vale la pena realizarlo para mejorar el asma.

\section{Estrategia de búsqueda:}

A través de la "Búsqueda mejorada en Pubmed" del sitio wwiv.infodoctor.org/rafabravo se emplearon las palabras "asthma" " reflux oesophagitis". Como buscábamos artículos sobre terapéutica limitamos a metanálisis o revisiones sistemáticas.

Esta estrategia de búsqueda arrojó 9 citas de las cuales una pareció la más apropiada para responder la pregunta.

\section{Resumen de la evidencia:}

Los autores de este trabajo ${ }^{1}$ realizaron una revisión sistemática de la literatura. Esta revisión fue realizada por la Cochrane Airways Group en 1998 y actualizada para la presente publicación. Se incluyeron todos los ensayos clínicos aletorizados (ECA) sobre tratamiento del RGE que reportaran algún resultado clínicamente relevante sobre asma.

Los estudios se identificaron a través de una búsqueda electrónica del Registro de ECA de la Colaboración Cochrane de ensayos publicados entre 1996 y abril de 2000. Además se realizaron contactos para obtener trabajos no publicados. Finalmente se incluyeron los CS y estudios controlados acerca de tratamiento del RGE en pacientes con asma.

Los estudios fueron evaluados por revisores independientes a fin de determinar su calidad metodológica.

Se incluyeron 12 estudios para la revisión. Cuatro estudios investigaron ranitidina o cimetidina, seis evaluaron inhibidores de la bomba de protones, uno evaluó el manejo conservador del RGE y otro tuvo tres ramas una de las cuales fue ranitidina, otra cirugía y otra placebo.

Características de los sujetos: los pacientes que se incluyeron en los estudios eran adultos con asma y RGE sintomático. La gravedad del asma fue desde leve hasta severa. EI RGE estaba confirmado por Phmetría, endoscopía, síntomas y manometría. Cuatro estudios reportaron asociación entre asma y RGE, uno de los cuales excluyó a los sujetos en quienes no se pudo demostrar asociación.

Duración del tratamiento: la duración fue entre una y seis semanas.

Medición de resultados: se evaluaron 432 sujetos de los cuales 396 completaron los ensayos. Los resultados fueron reportados inconsistentemente a través de los diferentes estudios lo que imposibilitó realizar un metanálisis.

Función pulmonar: no hubo un beneficio claro y contundente en el efecto del tratamiento antireflujo en el VEF 1. El flujo pico mejoró sólo en uno de los estudios.

Síntomas: los síntomas se midieron en todos los trabajos, pero de maneras tan diferentes que la cuantificación global de este resultado no fue posible.

- De los 12 ensayos, tres reportaron mejoría en los síntomas. Los otros 9 no encontraron mejoría en los síntomas.

- Seis ensayos reportaron síntomas nocturnos de asma. De éstos, 3 encontraron mejoría de los síntomas nocturnos con el tratamiento para RGE.

- Siete ensayos evaluaron el empleo de agonistas Beta2 en pacientes con tratamiento antireflujo, 3 de los cuales tres mostraron disminución en las dosis. El resto no mostró diferencias.

Conclusión de los autores: la literatura publicada no avala consistentemente al tratamiento del RGE como medio para controlar el asma. Se requiere la realización de ECA con mayor número de pacientes en los cuales se demuestre una relación temporal entre el RGE y el asma. Es necesario que los estudios que se realicen tengan al menos una duración de seis semanas para permitir tiempo suficiente de observación del posible efecto benéfico.

\section{Comentario:}

En esta revisión no se encontró evidencia consistente acerca de que el tratamiento del RGE sea beneficioso para pacientes con asma. No hubo efecto claro sobre la función pulmonar, ni sobre los síntomas o el empleo de medicación. Nueve de los doce trabajos reportaron al menos un resultado favorable, pero no hubo consistencia

No todos los sujetos presentaban RGE sintomático. Sólo en dos 
estudios se requirió la demostración de que el RGE precipitaba el asma.

Debemos reconocer que el número de sujetos enrolado en cada estudio fue pequeño y esto limitaría el poder de cada estudio para detectar diferencias entre los grupos. Los autores calcularon que sería necesario contar con 506 sujetos para detectar una diferencia de $20 \mathrm{ml} /$ minuto en el pico flujo con un poder de $80 \%$. El número de pacientes que entraron en estudios que midieron pico flujo fue de 184.

Hay que destacar que la duración de los estudios fue demasiado corta como para evaluar adecuadamente los eventos. Se podría especular que un período mayor de observación podría haber encontrado otros resultados.

La asociación entre RGE y asma parece ser más causal que casual a la luz de diferentes estudios.

Por último, cabe destacar que, para tener la mayor chance de encontrar mejoría en el asma, el tratamiento debería haber sido eficaz contra el RGE. En la presente revisión hubo diversos tratamientos (algunos de los cuales no son de elección en el manejo del RGE). Una pregunta obvia para realizarse es si los tratamientos administrados mejoraron el reflujo. Esto fue confirmado objetivamente en dos estudios que emplearon omeprazol y cirugía. Un diseño óptimo de un ensayo debería establecer que el RGE fue exitosamente tratado como requisito para valorar su efecto sobre el asma.

En el futuro, deberíamos contar con un ensayo que incluyera una rama con altas dosis de inhibidores de la bomba de protones y una rama con cirugía con un seguimiento de 6 meses. Los sujetos con asma y RGE en los cuales se demostrara que el RGE precipita episodios de asma deberían ingresar en el estudio. Habría que medir como resultados: síntomas, calidad de vida, función pulmonar y efectos del tratamiento sobre el RGE.

En resumen: el control del asma no mejora de manera consistente con el tratamiento del RGE. La mejoría de los síntomas fue más probable que la mejoría en la función pulmonar. Hasta el momento no podemos recomendar el tratamiento del RGE como medida universal para el control del asma. Se requiere mayor investigación en un grupo selecto con tratamiento médico o quirúrgico del RGE para a fin de establecer si el tratamiento del RGE mejora el asma.

Dra. Karin Kopitowski [ Unidad de Medicina Familiar y Preventiva. Hospital Italiano de Buenos Aires ]

Referencias

1- Coughlan JL. Gibson PG, Henry RL. Medical treatment for reflux does not consistently improve asthma control: a systematic review. Thorax 2001; 56: 198-204.
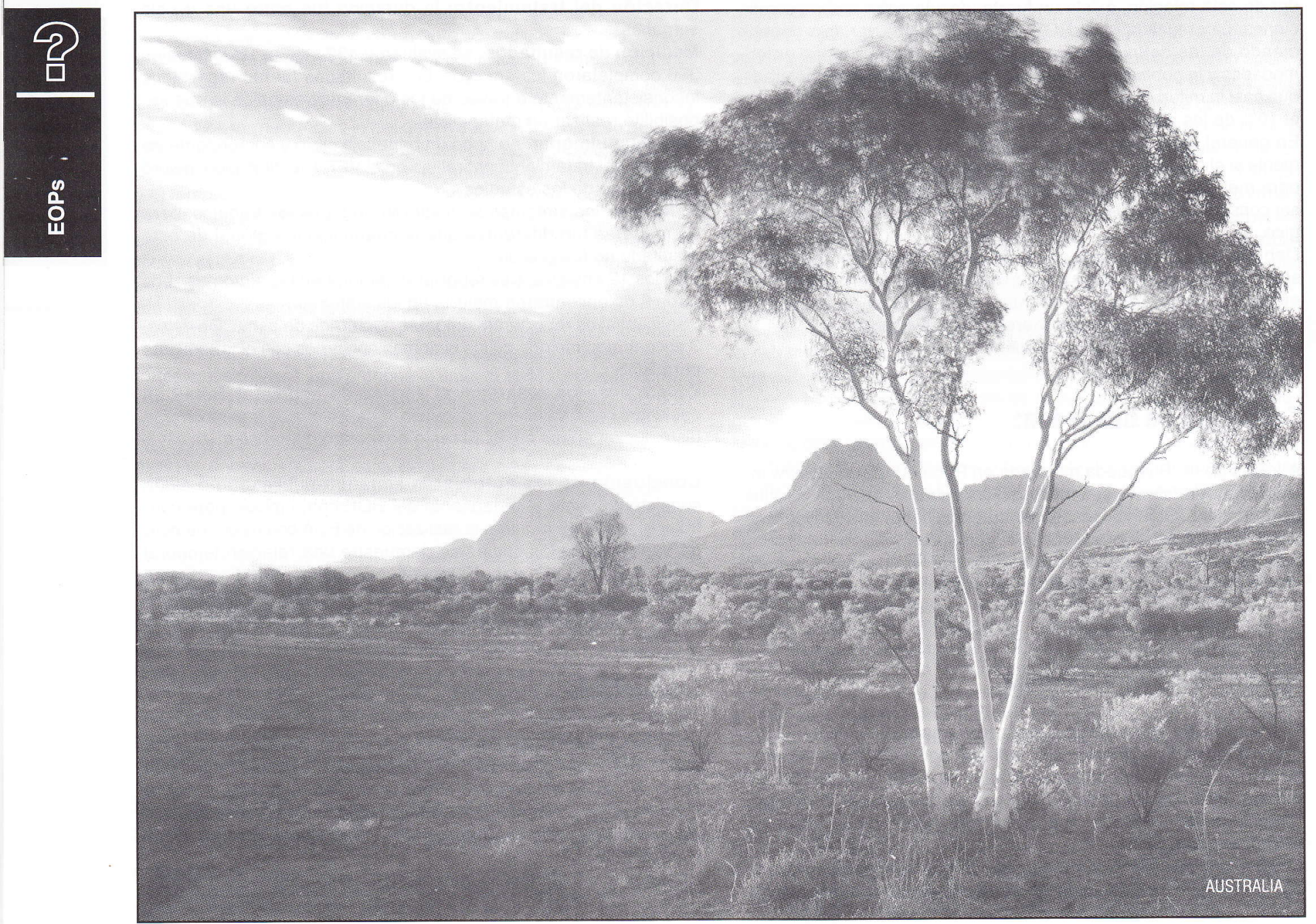